\title{
Spontaneous Subarachnoid Haemorrhage in a Case with Covid-19 Infection - Effect or Association? A Case Report
}

\author{
Sourya Acharya ${ }^{1}$, Samarth Shukla², Pankaj Banode ${ }^{3}$, Shefali Sharma ${ }^{4}$, Abhijeet Wadekar ${ }^{5}$ \\ 1, 5 Department of Medicine, Datta Meghe Institute of Medical Sciences (Deemed to Be University), \\ Sawangi, Wardha, Maharashtra, India. ${ }^{2}$ Department of Pathology, Datta Meghe Institute of Medical \\ Sciences (Deemed to Be University), Sawangi, Wardha, Maharashtra, India. ${ }^{3}$ Department of \\ Radiology, Datta Meghe Institute of Medical Sciences (Deemed to Be University), Sawangi, Wardha, \\ Maharashtra, India. ${ }^{4}$ Department of Radiology, Aster AI Raffah Hospital, Sohar, Oman.
}

\section{INTRODUCTION}

Covid-19 is a disease caused by the SARS-CoV-2 virus that usually causes mild flu-like illness in majority of the cases, but it can cause severe pneumonia and multiple organ dysfunction even death especially in elderly patients who also have comorbidities like hypertension, diabetes, chronic obstructive airway disease (COAD), asthma, and cardiac disease. The central and peripheral nervous systems are not spared, and neurological complications are frequently reported in severely ill patients who have comorbidities. The SARS-CoV-2 virus has the potential to invade the brain and it enters the brain via a haematogenous route or olfactory system through angiotensinconverting enzyme -2 receptors, present on endothelial cells of cerebral vessels. The most neurological manifestations, seen in Covid-19 infection are altered sensorium (agitation, delirium, and coma), ischemic or haemorrhagic stroke, acute disseminated encephalomyelitis or acute necrotizing encephalopathy, headaches, Guillain-Barré syndrome. Here is a case of a 70-year-old hypertensive female who presented to us with complaints of fever, headache and vomiting of 3 days duration and after investigations, a diagnosis of Covid -19 with hypertension and subarachnoid haemorrhage was made.

SARS-CoV-2 virus spreads through the haematogenous route, can disrupt the blood-brain barrier and enters into the brain. Evidence shows that it can travel transneuronally across the cribriform plate of the ethmoid bone and invade the brain via the olfactory nerve. ${ }^{1}$ Angiotensin-converting enzyme 2 receptors that are present on endothelial cells of cerebral vasculature act as cell entry points for the virus. ${ }^{1}$ In an autopsy-based study, the presence of SARS-CoV-2 virus in neural and capillary endothelial cells of the frontal lobe of the brain was reported. ${ }^{2}$ Comorbidities, like diabetes and hypertension, enhance the angiotensin-converting enzyme 2 receptor expression in the brain and neurotropism of the SARS-CoV-2 virus. ${ }^{3}$

\author{
Corresponding Author: \\ Dr. Sourya Acharya, \\ Professor and HOD, \\ Department of Medicine, \\ Datta Meghe Institute of Medical Sciences \\ (Deemed to be University, Sawangi, \\ Wardha, Maharashtra, India. \\ E-mail: souryaacharya74@gmail.com
}

DOI: $10.14260 /$ jemds $/ 2021 / 731$

How to Cite This Article:

Acharya S, Shukla S, Banode $P$, et al. Spontaneous subarachnoid haemorrhage (SAH) in a case with Covid-19 infectioneffect or association? a case report. J Evolution Med Dent Sci 2021;10(41):36073609, DOI: 10.14260/jemds/2021/731

Submission 12-05-2021,

Peer Review 21-09-2021,

Acceptance 28-09-2021,

Published 11-10-2021.

Copyright (C) 2021 Sourya Acharya et al. This is an open access article distributed under Creative Commons Attribution License [Attribution 4.0 International (CC $B Y 4.0)]$ 
Alteration in blood pressure control is another proposed mechanism that explains the increased risk of cerebral vascular complications. The ACE- 2 signalling lowers blood pressure. The competitive blockage of angiotensin-converting enzyme 2 by the SARS-CoV-2 virus down-regulates angiotensin-converting enzyme 2 expression leading to uncontrolled blood pressure that in turn increases the possibility of cerebrovascular accidents. ${ }^{4}$

In the medical literature, some case reports on the association of the Covid-19 infection and the occurrence of spontaneous subarachnoid haemorrhage (SAH) have been reported. This may be again due to the effect of the virus on blood pressure that contributes to SAH.

There is limited literature on the occurrence of SAH as a neurologic complication of Covid-19. Identifying factors for diagnosis and management of patients with Covid-19 infection and SAH is imperative.

\section{PRESENTATION OF CASE}

A 70-year-old female presented to us with chief complaints of high-grade fever of 3 days duration, continuous headache since 1 day and 4 episodes of vomiting over 12 hours. The fever was intermittent in nature without chills and rigours. The headache was predominantly occipital and nuchal and of intense severity. There were four episodes of vomiting associated. There was no history of diplopia, blurring of vision, convulsions, weakness, change of speech, cough, breathlessness, chest tightness, and haemoptysis.

On examination, the patient was anxious and restless. The examination was done under all Covid-19 protocols by the attending physician. Her fever was $101.8^{\circ} \mathrm{F}$; Pulse was 110/ minute, regular, Blood pressure- 192/110 mm of $\mathrm{Hg}$ in the right arm. SpO2 was $91 \%$ while breathing ambient air. JVP was normal. Quick cardiovascular and respiratory system examinations were normal. CNS examination: GCS- 15. Higher function and cranial nerves were intact. Power was grade 5/5 in all four limbs. There was no neck stiffness. Kernig's and Brudzinski's signs were negative. On admission, a noncontrast CT scan showed spontaneous SAH which was of grade 1 as per the modified Fisher scale. (Figure-1) Polymerase chain reaction (RT-PCR) and chest CT for Covid-19 were positive. Subsequent digital subtraction angiogram (DSA) examination did not reveal any aneurysm. (Figure-2, 3)

Investigations: $\mathrm{Hb}-12 \mathrm{gm} \%$, TLC- $9600 / \mathrm{mm} 3$ with lymphopenia ( 530 cells $/ \mathrm{mm}^{2}$ ), erythrocyte sedimentation rate of $85 \mathrm{~mm} / \mathrm{h}$, C-reactive protein- $3 \mathrm{mg} / \mathrm{L}$, creatinine of 1.1 $\mathrm{mg} / \mathrm{dL}$, platelets of $310 \times 10^{9} / \mathrm{L}$, prothrombin time of 11 seconds, international normalized ratio of 1 and partial thromboplastin time of 66 seconds, kidney and liver function tests were within the normal limits. Serum ferritin, D-dimer and lactate dehydrogenase were within normal limits.

She was treated with supplemental oxygen through nasal prongs, blood pressure was controlled by labetalol to an acceptable range of 140 systolic and $90 \mathrm{~mm}$ of $\mathrm{Hg}$ diastolic, tab. nimodipine $60 \mathrm{mg}$ per oral every 4 hourly, IV antibiotics and multivitamins were prescribed. Her general condition improved, headache and fever subsided after 5 days and she was discharged and was advised home isolation.
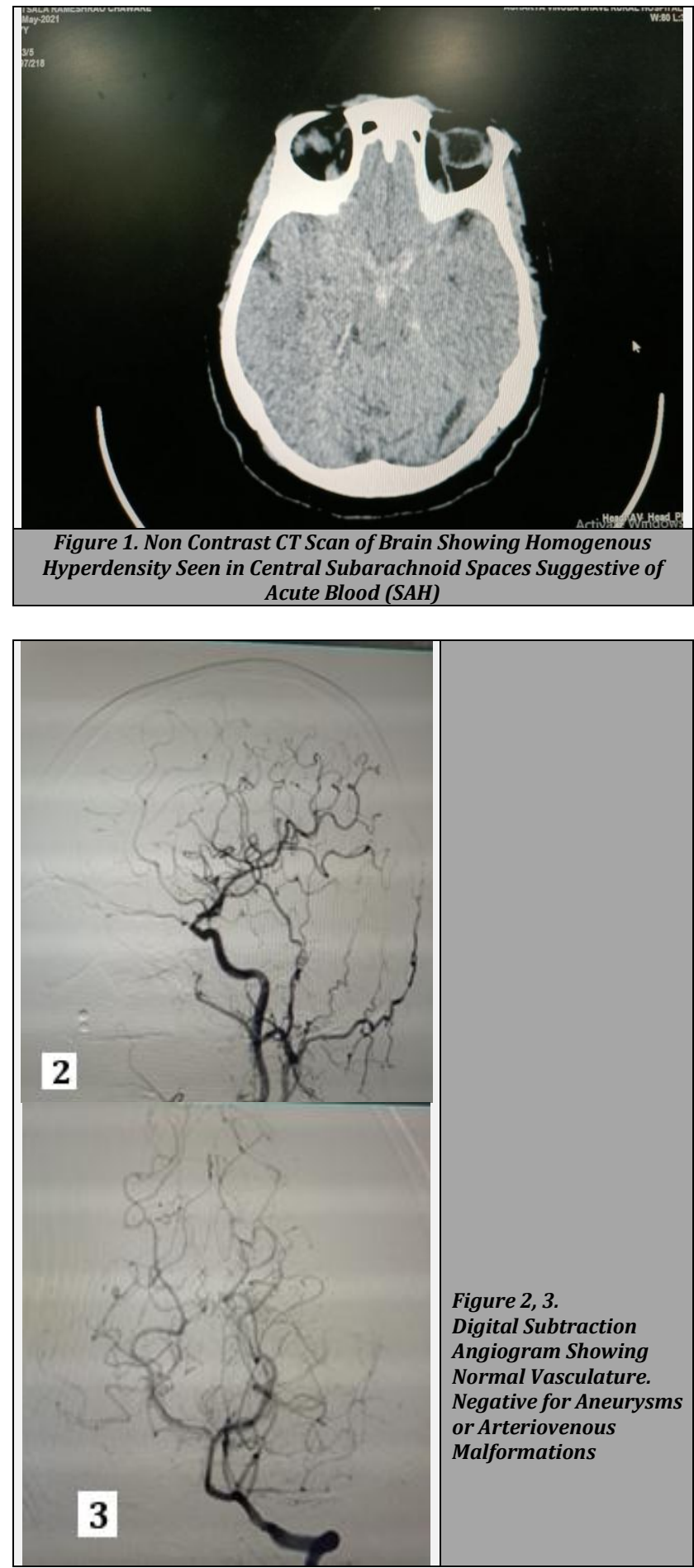

DISCUSSION

Spontaneous SAH can be a direct or indirect effect of Covid-19. The presumed cause may be endothelial dysfunction associated. A study conducted by Reddy et al. reported 12 cases with Covid-19 infection and concurrent cerebrovascular disease out of which two cases were SAH. ${ }^{5}$ In a European multicentre study, out of eighteen Covid-19 patients, 11 (61.1 $\%$ ) had SAH, but secondary SAH was seen in only one patient. In this European multicenter study case series, no aneurysm was found in DSA. 
The major pathologic state of necrotizing vasculitis is typically associated with parenchymal haemorrhage. ${ }^{6}$ Apart from endothelial dysfunction, other factors that can predispose for the development of SAH in Covid-19 patients can be thrombocytopenia, anticoagulant medications, extracorporeal membrane oxygenation (ECMO) and mechanical ventilation. ${ }^{6}$ Our patient did not have any of the above risk factors except hypertension which usually causes intracerebral bleeding.

\section{CONCLUSIONS}

Spontaneous SAH should be suspected when any patient with positive Covid-19 status presents with headaches. An urgent CT scan of the brain is mandatory to rule out this potentially catastrophic complication. Conventional management with oral nimodipine is essential to prevent vasospasm and subsequent strokes along with protocol-based Covid-19 treatment. In this current pandemic, every suspected SAH patient should be screened for possible Covid-19 symptoms also.

Financial or other competing interests: None.

Disclosure forms provided by the authors are available with the full text of this article at jemds.com.

\section{REFERENCES}

[1] Li MY, Li L, Zhang Y, et al. Expression of the SARS-CoV-2 cell receptor gene ACE2 in a wide variety of human tissues. Infect Dis Poverty 2020;9(1):45.

[2] Paniz-Mondolfi A, Bryce C, Grimes Z, et al. Central nervous system involvement by severe acute respiratory syndrome coronavirus-2 (SARS-CoV-2). J Med Virol 2020;92(7):699-702.

[3] Puelles VG, Lütgehetmann M, Lindenmeyer MT, et al. Multiorgan and renal tropism of SARS-CoV-2. N Engl J Med 2020;383(6):590-592.

[4] Zhu H, Rhee JW, Cheng P, et al. Cardiovascular complications in patients with COVID-19: Consequences of viral toxicities and host immune response. Curr Cardiol Rep 2020;22(5):32.

[5] Reddy ST, Garg T, Shah C, et al. Cerebrovascular disease in patients with covid-19: a review of the literature and case series. Case Rep Neurol 2020;12(2):199-209.

[6] Nawabi J, Morotti A, Wildgruber M, et al. Clinical and imaging characteristics in patients with SARS-CoV-2 infection and acute intracranial hemorrhage. J Clin Med 2020;9(8):2543. 